1 Organização Mundial da Saúde (OMS). Organização Pan-Americana da Saúde (Opas) - Brasília (DF), Brasil.

rodrigujos@paho.org

2 Ministério da Saúde (MS)

- Brasília (DF), Brasil.

3 Universidade do Estado do Rio Grande do Norte (UERN) - Mossoró (RN) Brasil.

4 Universidade de São Paulo (USP) - São Paulo (SP), Brasil.

\section{Educação Interprofissional nas políticas de reorientação da formação profissional em saúde no Brasil}

\author{
Interprofessional Education in the policies of reorientation of \\ professional training in health in Brazil \\ José Rodrigues Freire Filho', Cláudia Brandão Gonçalves Silva², Marcelo Viana da Costa ${ }^{\mathbf{2}}$, Aldaísa \\ Cassanho Forster 4
}

DOI: 10.1590/0103-11042019S107

RESUMO O processo de formação dos profissionais de saúde encontra-se no momento histórico de reformas orientado para o fortalecimento dos sistemas de saúde, com grandes desafios para esse novo século. Iniciativas para a superação dos problemas relacionados com a colaboração entre diferentes profissionais de saúde são assumidas como uma das prioridades. É partindo da necessidade de formar profissionais de saúde mais aptos à colaboração para o trabalho em equipe que a Educação Interprofissional (EIP) começa a ser valorizada em todo o mundo. O objetivo deste ensaio foi apresentar a trajetória histórica da incorporação dessa abordagem nas políticas de formação profissional em saúde do Brasil, bem como destacar os recentes avanços nessa direção. Recentemente, o Brasil passou a incorporar a EIP de forma mais clara nas políticas de reorientação da formação em saúde, processo intensificado nos últimos dois anos, o que pode ser atribuído ao reconhecimento dos formuladores de políticas para a importância da abordagem no processo de formação profissional em saúde no País, assim como à atuação de organismos internacionais de saúde que intensificaram o chamado para a sua relevância no processo de mudança do modelo de atenção à saúde.

PALAVRAS-CHAVE Relações interprofissionais. Recursos humanos. Política de saúde. Planejamento em saúde. Educação em saúde.

ABSTRACT The process of training of health professionals is set in the historical moment of reforms oriented towards the strengthening of health systems, with great challenges for this new century. Initiatives to overcome problems related to collaboration among different health professionals are taken as one of the priorities. Starting from the need to train health professionals better able to collaborate in teamwork, Interprofessional Education (IPE) begins to be valued throughout the world. The objective of this essay is to present the historical trajectory of the incorporation of such approach in the policies of professional training in health in Brazil, as well as to highlight the recent advances in this direction. Brazil has recently begun to incorporate IPE more clearly into policies for reorienting health education, a process intensified in the last two years, which can be attributed to the recognition of policymakers for the importance of the approach in the training process in health care in the country, as well as the work of international health organizations that have intensified the call for their relevance in the process of change of the health care model.

KEYWORDS Interprofessional Relations. Human resources. Health policy. Health planning. Health Education. 


\section{Introdução}

O processo de formação profissional em saúde encontra-se no momento histórico de reformas orientado para o fortalecimento dos sistemas sanitários, com grandes desafios para esse novo século. Nesse contexto, um dos mais expressivos obstáculos consiste em atender às complexas e dinâmicas necessidades de saúde, tendo em vista que a reforma do modelo de atenção precisa vir acompanhada de um processo sólido de reorientação do modelo de formação, em um movimento de interdependência ${ }^{\mathbf{1}, \mathbf{2}}$.

O deficit e a distribuição desigual de profissionais de saúde somam-se a modelos de formação pouco alinhados aos princípios necessários ao fortalecimento dos sistemas de saúde, com pouca capacidade de formação de perfis adequados às necessidades de saúde ${ }^{\mathbf{3 , 4}}$.

A sociedade no atual contexto experimenta uma tripla carga de necessidades e problemas de saúde: transformações demográficas e epidemiológicas e a incorporação de agravos externos típicos de mudanças sociais e culturais. Todo esse cenário desvela que não é possível atender às necessidades de saúde - cada vez mais complexas - de forma efetiva a partir de modelos de produção de serviços de saúde fragmentados. Ao contrário, requer um novo perfil profissional, mais apto a ofertar serviços de saúde integrais, com maior incorporação e valorização da colaboração como princípio orientador do processo de trabalho em saúde ${ }^{5-7}$.

Destarte, iniciativas para superação dos problemas relacionados com a colaboração entre os diferentes profissionais e maior integração entre os serviços de saúde de diferentes níveis de complexidade são assumidas como uma das prioridades; isto porque uma força de trabalho em saúde integrada e disposta a atuar conjuntamente está diretamente associada à melhoria da assistência em saúde $\mathbf{8}^{\mathbf{8} 9}$.

Partindo da necessidade de formar profissionais de saúde mais aptos à colaboração e com competências para a execução do efetivo trabalho em equipe é que a Educação
Interprofissional (EIP) começa a ser valorizada em todo o mundo. Definida como a ocasião em que membros de duas ou mais profissões aprendem em conjunto, de forma interativa, com o propósito explícito de melhorar a colaboração e qualidade da atenção à saúde, a EIP apresenta marcos teórico-conceituais e metodológicos capazes de ajudar no processo de reorientação da formação e do trabalho em saúde, com vistas à superação de importantes gargalos da realidade da produção dos serviços de saúde 9 .

No contexto mundial, a sistematização do debate sobre a EIP é relativamente recente. Alguns autores atribuem os primeiros movimentos a um grupo de experts da Organização Mundial da Saúde (OMS) no início da década de 1980. Outros, por sua vez, apresentam a década de 1960, no Reino Unido, como a gênese dos primeiros movimentos de sistematização de seus pressupostos, sendo fortemente atrelados à discussão sobre a necessidade de encontrar estratégias educacionais que pudessem superar a histórica fragmentação do trabalho em saúde e suas implicações na qualidade da atenção à saúde e à segurança dos pacientes ${ }^{10}$.

Os primeiros esforços foram estimulados a pensar formas de enfrentar uma grande incoerência: profissionais que necessariamente precisavam trabalhar juntos estavam sendo formados separados. A tendência em formar profissionais, com ênfase no desenvolvimento de competências específicas, conseguiu, ao longo da história, legitimar o que a literatura define como silos profissionais ou tribalismo das profissões. Esse modelo de formação sustenta práticas em saúde fortemente fragmentadas, com exposição do usuário a atos duplicados, o que leva ao retrabalho dos profissionais de saúde, aumentando os riscos de erros e custos em saúde ${ }^{10}$.

Reconhecendo a potência da EIP no movimento global de mudança na lógica de formação em saúde, a OMS realiza importantes iniciativas para valorização desse debate, lançando publicações que exercem importante papel de orientação dos formuladores de políticas de saúde e educação para 
a incorporação dos pressupostos teórico-conceituais e metodológicos da EIP9,11.

A literatura apresenta importantes evidências sobre o papel da EIP no enfrentamento dos problemas relacionados com os modelos hegemônicos de formação e de atenção em saúde, consolidando-se enquanto abordagem educacional, com atributos que fortalecem os sistemas de saúde, na medida em que utiliza metodologias de ensino-aprendizagem para desenvolver competências profissionais de comunicação, interação e colaboração profissional ${ }^{5,8,11-14}$.

Tomando por base esse panorama, esse ensaio adotou como objetivo apresentar aspectos que demonstram a trajetória histórica de incorporação da EIP nas políticas de formação profissional em saúde do Brasil e os avanços nessa direção, a partir de referenciais que destacam que essa abordagem preconiza a interação entre estudantes ou profissionais de diferentes profissões, por meio de processos de aprendizagem compartilhados e significativos e que tenham como clara finalidade o desenvolvimento de competências profissionais colaborativas.

\section{A EIP nas políticas de formação em saúde no Brasil}

Ao considerar que a educação tradicional e as práticas uniprofissionais apresentam limitações que obstam o efetivo atendimento das complexas necessidades de saúde, bem como a operacionalização dos princípios adotados pelo Sistema Único de Saúde (SUS), a EIP ganha destaque por ser dotada de elementos capazes de inverter a lógica da educação verticalizada, com vistas a promover o aprendizado compartilhado, o que oportuniza avanços para o processo de trabalho das equipes de saúde presentes na realidade brasileira, ${ }^{5,12}$.

No Brasil, o debate sobre o trabalho em equipe não é recente. Desde os primeiros movimentos em torno do processo de construção do
SUS, o trabalho em equipe se apresenta como prerrogativa para a implantação de um novo sistema de saúde, fundado sob os princípios da universalidade, equidade e integralidade. Entretanto, no que se refere ao processo de formação dos profissionais de saúde, a incorporação de aspectos educacionais capazes de desenvolver competências para trabalhar em conjunto ainda ocorre de forma tímida ${ }^{\mathbf{2}}$.

Produções científicas nacionais apresentam relatos de importantes iniciativas de aprendizagem compartilhada entre estudantes de diferentes cursos da saúde, mas que ainda carecem de elementos que demonstrem a intencionalidade em desenvolver competências colaborativas nesse processo. Reunir estudantes de diferentes profissões da área da saúde em um mesmo espaço não é suficiente para afirmar que se trata de uma iniciativa assentada na proposta da EIP. Há a necessidade de que o processo de encontro seja potencializado por metodologias que valorizem a colaboração e consiga explorar conhecimentos, habilidades, atitudes e valores capazes de sustentar uma cultura de formação e trabalho, ancoradas na centralidade do usuário, na interação permanente e interdependência entre as diferentes práticas profissionais $^{12-14}$.

Cumpre destacar que o Brasil tem um histórico importante de políticas implementadas com vistas à superação do modelo biomédico e individual, com destaque para a educação baseada em competências; maior integração entre universidade, serviços de saúde e comunidade; e mudanças curriculares com importante incorporação de métodos ativos de aprendizagem e maior protagonismo dos estudantes ${ }^{\mathbf{1 2}, \mathbf{1 3}}$.

Entre essas políticas, o Programa UNI - Uma Nova Iniciativa na Educação dos Profissionais do Setor de Saúde, da década de 1990, foi um marco inicial importante na medida em que se propôs uma mudança de paradigma na educação desses profissionais a partir da crítica ao modelo flexneriano de formação médica, com incorporação da perspectiva interdisciplinar 
e multidisciplinar, com abordagens que se aproximam dos marcos teórico-conceituais e metodológicos da EIP ${ }^{15}$.

A Política Nacional de Educação Permanente em Saúde (PNEPS), instituída em 2004, também se configura como importante política de reorientação das práticas e da formação em saúde por trazer em suas diretrizes importantes elementos que direcionam ações e programas para a integração entre profissionais, universidades, gestores e controle social em um amplo processo educativo, tendo como lócus privilegiado o cotidiano do trabalho em saúde. São aspectos que desenham um cenário adequado para a incorporação e fortalecimento de metodologias e teorias capazes de orientar o processo de desenvolvimento das competências colaborativas assentadas na lógica da EIP15,16. Historicamente, a PNEPS vem se consolidando com uma das políticas mais potentes para a transformação das práticas e da formação em saúde. Entretanto, ainda se faz necessário maior sistematização e clareza na intencionalidade na incorporação dos pressupostos da EIP nas estruturas, ações e programas que compõem essa política.

Outro marco nacional na política de educação e saúde do Brasil foi a instituição do Fórum Nacional da Educação das Profissões da Área da Saúde (FNEPAS), criado no ano de 2004. O FNEPAS consiste em um importante espaço conduzido por uma rede de associações profissionais da área da saúde, cujo objetivo é discutir sobre temas para a contribuição na construção de cenários institucionais às mudanças na formação em saúde. Por sua estrutura e composição, com representantes de todo o País, esse fórum se configura como espaço de grande relevância para a ampliação do debate da EIP, tendo em vista que, ao longo da história, sempre incorporou lutas relevantes no processo de fortalecimento e consolidação do SUS ${ }^{15,16}$.

Outras iniciativas - em anos mais recentes - apresentam o tema da interprofissionalidade como um dos eixos de mudança na formação em saúde de forma mais clara, a exemplo do Programa de Educação pelo Trabalho para a
Saúde (PET-Saúde), instituído pelos Ministérios da Saúde e da Educação, no ano de $2008^{17}$. O PET-Saúde tem como alvo as Instituições de Ensino Superior (IES), visando estimular o desenvolvimento de ações de interação e comunicação entre os diferentes cursos de graduação, por meio da inserção dos estudantes nos serviços de saúde, possibilitando a experiência do trabalho coletivo com ênfase na integração ensino-serviço. Reconhece-se que o programa trouxe avanços para o debate da EIP no Brasil, porém os resultados demonstram que as instituições de ensino necessitam de maior suporte para dar sustentabilidade às ações desenvolvidas nessa perspectiva e que é preciso legitimar o ensino interprofissional nas políticas de reorientação da formação em saúde14,17.

Contribuições de organismos internacionais também foram relevantes para influenciar a incorporação da EIP na política de educação e saúde do Brasil. No ano de 2010, a OMS publicou o Marco para a Ação em Educação Interprofissional e Prática Colaborativa, com tradução para o português, o qual exerceu importante papel de divulgação dos pressupostos da EIP no País. O conteúdo da publicação é expressivo e demarca o forte compromisso da organização para com o fortalecimento desse tema por meio de recomendações nas políticas de formação profissional em saúde, incluindo a EIP em sua agenda de prioridades ${ }^{18}$.

Outro movimento importante consistiu na incorporação da EIP em importantes bases legais da educação no âmbito da graduação. As Diretrizes Curriculares Nacionais (DCN) para os cursos de graduação em medicina, publicadas no ano de 2014, demonstram a presença da EIP na dimensão macro da realidade, com possibilidades de induzir mudanças nas dimensões meso e micro' ${ }^{19}$. Na referida normativa, encontra-se explícito que o processo de ensino-aprendizagem do futuro profissional médico deverá contemplar o desenvolvimento de competências para o trabalho em equipe, voltadas para a integração e interprofissionalidade ${ }^{20}$.

Iniciativas pautadas na EIP aparecem nos 
currículos de IES dos estados do Ceará, do Rio Grande do Sul, do Rio Grande do Norte, do Rio de Janeiro, de Minas Gerais, de São Paulo e do Distrito Federal, sendo a maior parte delas voltadas aos processos de integração ensino-serviço-comunidade, em articulação com a atenção básica. Essas experiências, inovadoras em seus processos educacionais relacionadas com a viabilização de espaços de aprendizagem compartilhadas, demonstram a potência da realidade brasileira em fortalecer o ensino baseado na interprofissionalidade, não apenas na graduação, mas também na pós-graduação ${ }^{16}$.

Verifica-se que, em anos mais recentes, houve ampliação do número de publicações e pesquisas relacionadas com a incorporação da EIP em diferentes contextos do País ${ }^{12}$, as quais vêm confirmando que, no âmbito do processo de trabalho em saúde, avançou-se no que se refere à adoção dos pressupostos teórico-metodológicos da EIP a partir de ações de educação permanente. Processo facilitado pela própria natureza e conformação do trabalho em saúde do SUS, desenvolvido por meio de arranjos multiprofissionais, que viabilizam processos educacionais com ênfase na interprofissionalidade ${ }^{\mathbf{1 2 , 1 3}}$.

Não obstante, cumpre ratificar que a utilização dessa abordagem no ensino na saúde ainda é incipiente e de frágil suporte teórico, circunstância que imprime a necessidade de elaborar estratégias de apoio aos processos de implementação da EIP no contexto brasileiro $^{12,15,21}$.

\section{Plano de ação brasileiro para a implementação da EIP}

Dezembro de 2016 pode ser considerado um marco para a EIP na região das Américas, a partir da atuação ativa da Organização PanAmericana da Saúde (Opas), que convocou seus Estados Membros a conhecerem e debaterem sobre o tema da EIP em uma reunião técnica realizada na cidade de Bogotá, Colômbia, na qual encorajou os países a elaborarem um plano de ação nacional para a implementação dessa abordagem ${ }^{22}$.

A partir do ano de 2017, motivado pela agenda proposta pela Opas, o Ministério da Saúde do Brasil priorizou a inclusão da pauta da EIP em suas estratégias para o fortalecimento da política de formação profissional em saúde. De forma imediata, promoveu articulações com o Ministério da Educação (MEC), IES e a Rede Brasileira de Educação e Trabalho Interprofissional em Saúde (ReBETIS) para a elaboração do plano nacional ${ }^{23}$.

A estruturação do plano partiu do levantamento das principais iniciativas educacionais em andamento no País com potencial para aplicação dos pressupostos teóricos e metodológicos da EIP, levando em conta o arcabouço organizacional do SUS.

Após debates coletivos, o plano de ação brasileiro para a implementação da EIP, organizado em cinco linhas de ação descritas na figura 1, foi pactuado para o biênio 2017-2018. 
Figura 1. Linhas de ação do plano brasileiro para a implementação da EIP (2017-2018)

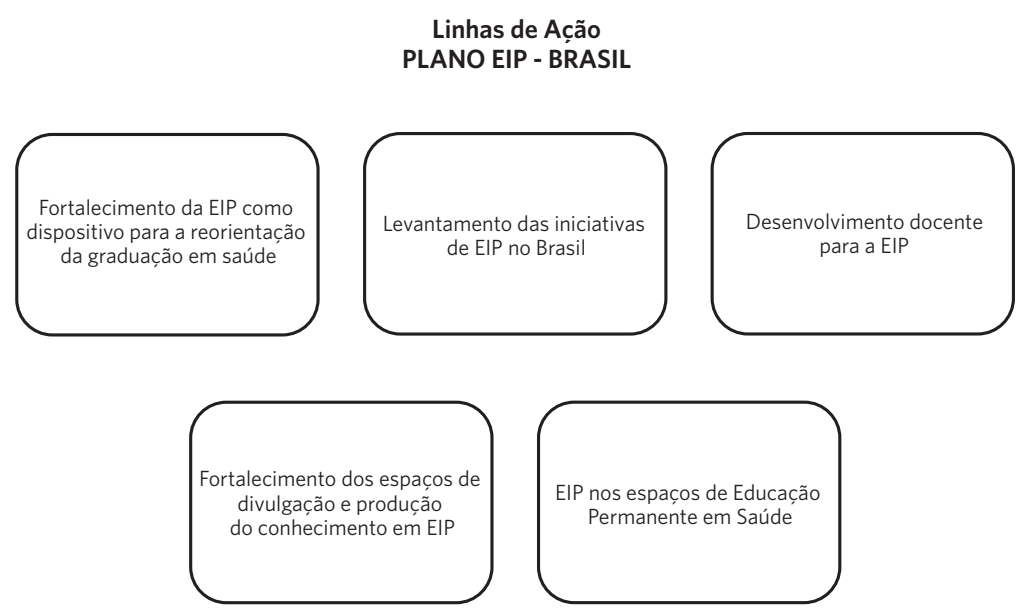

Fonte: Brasil, 201724

\section{Linha de ação 1: Fortalecimento da EIP como dispositivo para a reorien- tação dos cursos de graduação em saúde}

Com a finalidade de apoiar as novas escolas de medicina, criadas no âmbito do Programa Mais Médicos (PMM), a incorporar os pressupostos da EIP em coerência com as novas DCN para os cursos de graduação em medicina, a primeira linha de ação do plano previu a realização de oficinas regionais com a participação de um representante de cada IES com novo curso de medicina. O objetivo das oficinas foi debater e instrumentalizar as escolas no aprofundamento das bases teórico-metodológicas da EIP. O conjunto de eventos, realizado ao longo de 2017 e 2018, possibilitou realizar um levantamento das principais necessidades e desafios para adoção da EIP nas IES brasileiras, além de identificar sugestões que pudessem fortalecer o uso dessa abordagem no processo de formação em saúde no Brasil. Esse processo foi iniciado com os cursos de medicina em razão da publicação das DCN de 2014, já que foram as primeiras da área da saúde a conter orientações explícitas relacionadas com a EIP, em consonância com as mudanças da educação médica no País, conforme preconizado pelo $\mathrm{PMM}^{24,25}$. Apesar disso, representantes de outros cursos da saúde foram convidados para as oficinas como forma de fomentar o debate para o avanço da EIP nos diferentes cenários.

Outra atividade presente na primeira linha de ação do plano foi o debate e discussões realizadas com a Comissão Intersetorial de Recursos Humanos e Relações do Trabalho/ Conselho Nacional de Saúde (CIRHRT/CNS), para que o tema da EIP fosse inserido, de forma enfática, nas DCN dos demais cursos de graduação da área da saúde. Como resultado, foi publicada a Resolução CNS no 569 , de 8 de dezembro de 2017, que aprova o Parecer Técnico $n^{\circ} 300 / 2017$, o qual apresenta princípios gerais a serem incorporados nas DCN de todos os cursos de graduação da saúde, como elementos norteadores para o desenvolvimento dos currículos e das atividades didático-pedagógicas com enfoque na EIP26.

\section{Linha de ação 2: Levantamento das iniciativas de EIP no Brasil}

Um levantamento nacional de experiências de EIP, como forma de responder a importantes questões desconhecidas sobre o uso dessa 
abordagem no País, também foi uma atividade expressa no plano de ação. Organizado sob a forma de investigação e desenvolvido em duas fases, o levantamento buscou responder aos seguintes questionamentos: Quais IES brasileiras apresentam iniciativas de EIP? Como se configuram/caracterizam essas iniciativas? Quais as competências (conhecimentos, habilidades, atitudes e valores) necessárias para o desenvolvimento docente e para o fortalecimento das inciativas existentes e para a implantação de novas intervenções de EIP no País?24.

Por meio de survey descritivo-exploratório, buscou-se identificar e analisar o cenário para a implantação da EIP, considerando os limites, potências e características das iniciativas (organização acadêmica, organização administrativa, organização da matriz curricular, caráter obrigatório ou eletivo da iniciativa de EIP, existência de disciplinas, módulos e componentes curriculares de EIP ministrados, iniciativas de EIP como atividades de extensão e de pesquisa, metodologias de ensino e de avaliação adotadas e participação da IES em políticas indutoras de EIP) ${ }^{24}$.

\section{Linha de ação 3: Desenvolvimento docente para a EIP}

No âmbito da terceira linha de ação, foi ofertada uma ação formativa a professores e gestores dos cursos de graduação em saúde e a preceptores da área da saúde, com carga horária total de 80 horas, na modalidade de Ensino a Distância (EaD). Objetivou-se qualificá-los para a adoção dos princípios da EIP, nos cursos da saúde, como abordagem capaz de formar profissionais mais aptos ao trabalho interprofissional e colaborativo, além de encorajá-los para o desenvolvimento de iniciativas orientadas pelas bases teórico-conceituais e metodológicas da EIP, observando as especificidades das IES, serviços de saúde e suas diversidades regionais e culturais ${ }^{24}$. O curso foi concluído por 192 atores de todas as regiões do País.

\section{Linha de ação 4: Fortalecimento dos espaços de divulgação e produção do conhecimento em EIP}

A chamada pública para submissão de artigos sobre EIP e trabalho interprofissional em saúde no Brasil à 'Revista Interface: Comunicação, Saúde, Educação' compôs outra atividade do plano. A chamada teve como finalidade estimular a produção de conhecimentos e experiências sobre o tema e fomentar sua difusão nacional e internacional. Como resultado, foram recebidas 160 submissões de grande relevância para o cenário do trabalho e da formação em saúde no Brasil, sendo selecionados 19 artigos originais, quatro relatos de experiência e um debate. A maioria das submissões abordou temas e realidades do processo de EIP, mas também foi marcante o número de produções relacionadas com o trabalho e com as práticas interprofissionais em saúde ${ }^{27}$.

Outra atividade desenvolvida foi a realização da II Reunião Técnica Regional sobre EIP: Melhorar a Capacidade dos Recursos Humanos para Avançar a Saúde Universal, entre os dias 5 e 6 de dezembro de 2017, na cidade de Brasília, DF. O evento, que contou com 120 participantes, reuniu representantes de aproximadamente 30 países da América Latina e Caribe, bem como representantes do Canadá, Reino Unido, Espanha e de países da África, e teve como objetivo aprofundar o debate sobre o tema da EIP e apresentar experiências práticas para sua implementação; discutir os seus processos de implementação nas políticas de formação profissional em saúde dos países e os desafios que enfrentam para a integração dos setores da educação e da saúde; e estabelecer uma agenda comum para fortalecer a EIP na Região das Américas e discutir as diretrizes de ação que orientam os países a implementar atividades para expandir o seu uso na Região das Américas ${ }^{28}$.

Os acordos da II Reunião foram: o estabelecimento de uma ampla agenda para a incorporação do tema da EIP nas políticas de educação 
na saúde dos países da Região das Américas e a formalização de uma rede para potencializar as experiências de EIP entre os países - a Rede Regional de Educação Interprofissional das Américas (Reip) que tem o Brasil como representante da secretaria executiva para o período 2018-2021, juntamente com a Argentina e com o Chile 28 .

Para o Brasil, o evento representou um marco histórico no campo das políticas de formação em saúde, porque possibilitou o reconhecimento, em âmbito internacional, da EIP como prioridade para a transformação do modelo de atenção à saúde, da correspondência com os princípios do SUS e do seu potencial para melhoria da atenção à saúde.

No conjunto das atividades para o fortalecimento e divulgação da EIP, previsto no plano do Brasil, o Ministério da Saúde também apoia eventos organizados pela ReBETIS, conformada no ano de 2015. Essa rede realiza colóquios anuais e discute importantes aspectos relacionados com a pesquisa e com o desenvolvimento das práticas colaborativas e da EIP, tanto em nível nacional quanto internacional, com o objetivo de fortalecer a proposta no País ${ }^{24}$.

\section{Linha de ação 5: EIP nos espaços de Educação Permanente em Saúde}

Como proposta para inclusão da EIP nos espaços de Educação Permanente em Saúde (EPS), privilegiaram-se os processos formativos dos médicos do PMM, por considerar que a incorporação desse profissional na atenção básica, preenchendo uma lacuna histórica de escassez desse profissional nas equipes, pode potencializar o desenvolvimento de práticas colaborativas por meio da EIP. A oferta de um curso on-line sobre EIP foi um dos resultados das ações do plano, cuja finalidade consistiu em ampliar as competências para o trabalho colaborativo dos profissionais médicos e demais profissionais de saúde inseridos no projeto ${ }^{29}$.

Nessa linha de ação, também cabe mencionar o lançamento do Laboratório de Inovação em Educação na Saúde, no mês de setembro de 2017, que em sua primeira edição teve por tema a EPS e contemplou entre os seus eixos temáticos a EIP e as práticas interprofissionais. Essa atividade justificou-se pela necessidade de fortalecer a interlocução entre a EIP e a PNEPS enquanto dispositivo que dialoga com as bases dos sistemas educacional e de saúde brasileiros. O laboratório recebeu a inscrição de 251 experiências, sendo 92 relacionadas com a EIP. Dessas, três foram reconhecidas como propostas contribuintes para a implementação de práticas interprofissionais no País ${ }^{30}$.

O plano de ação conseguiu avançar em pontos estratégicos para o fortalecimento da EIP no contexto da formação e do trabalho em saúde, tais como o desenvolvimento docente para a EIP, o mapeamento das iniciativas de EIP nas IES brasileiras, o fortalecimento dos espaços de divulgação e produção do conhecimento em EIP e o estímulo a ações que visam dar concretude à EIP e práticas interprofissionais nos espaços de EPS ${ }^{\mathbf{2 8}}$.

No ano de 2018, outro grande passo foi dado com o objetivo de impulsionar o alinhamento dos cursos de graduação em saúde aos marcos teórico-conceituais e metodológicos da EIP: o lançamento do edital do PET-Saúde/Interprofissionalidade. Foram selecionados 120 projetos de IES públicas e privadas sem fins lucrativos de todas as regiões do Brasil, com a intencionalidade de estabelecer um processo permanente de acompanhamento, apoio e avaliação das ações de EIP com vistas ao seu fortalecimento e difusão e, consequentemente, aumento da qualidade da atenção à saúde ${ }^{31}$.

\section{Conclusões}

Apenas recentemente o Brasil vem incorporando de forma mais clara a EIP nas políticas de reorientação da formação em saúde, embora já se contabilizem iniciativas potentes para o seu desenvolvimento e consolidação de suas bases teóricas e metodológicas9,32,33. Esse aspecto foi intensificado nos últimos dois anos, o que 
pode ser atribuído ao reconhecimento e sensibilização dos formuladores de políticas quanto à importância do tema no processo de formação dos profissionais de saúde e seus potenciais efeitos sobre o modelo de atenção à saúde, bem como à atuação de organismos internacionais de saúde que intensificaram o chamado para o debate acerca da EIP, possibilitando demonstrar os benefícios dessa abordagem.

Muitos desafios ainda estão previstos nesse horizonte, pois mesmo que haja espaço adequado para o fortalecimento da EIP nas políticas existentes, há de se pensar em estratégias que assegurem a sustentabilidade das iniciativas no contexto histórico de enfrentamento dos desafios relacionados com a formação em saúde. A importância do tema, complexidade e desafios evidenciam a necessidade de maior investimento nessa proposta, com vistas a induzir mudanças sólidas e promissoras no contexto das instituições de ensino e saúde e, acima de tudo, no que diz respeito às relações profissionais.

\section{Colaboradores}

Freire Filho JR (0000-0003-1306-9368)* contribuiu para a concepção, planejamento e elaboração do rascunho, revisão crítica do conteúdo e aprovação final do manuscrito. Gonçalves CB (0000-0002-7644-9440)* participou substancialmente do planejamento, revisão crítica do conteúdo e da aprovação da versão final do manuscrito. Costa MV (0000-0002-3673-2727)* contribuiu para a concepção, planejamento, elaboração do rascunho, revisão crítica do conteúdo e aprovação final do manuscrito. Forster AC (0000-00022720-5802)* contribuiu para a concepção e planejamento da proposta, revisão crítica e aprovação da versão final. 


\section{Referências}

1. Portela GZ, Fehn AC, Ungerer RL, et al. Recursos humanos em saúde: crise global e cooperação internacional. Ciênc. Saúde Colet. 2017; 22(7):2237-2246.

2. Organização Mundial da Saúde, Organização PanAmericana da Saúde. Estratégia de Recursos Humanos para o Acesso Universal à Saúde e a Cobertura Universal de Saúde. 29a Conferência Sanitária PanAmericana, 69ª Sessão do Comitê Regional da OMS para as Américas; 25-29 set. 2017. Washington, DC: OPAS; 2017.

3. Reeves S, Perrier L, Goldman J, et al. Interprofessional education: effects on professional practice and healthcare outcomes (update). Cochrane Database Syst Rev. 2013; 28(3):CD002213.

4. Silva RP, Barcelos AC, Hirano BQL, et al. A experiência de alunos do PET-Saúde com a saúde indígena e o programa mais médicos. Interface (Botucatu). 2015; 19(supl1):1005-1014.

5. Matuda CG, Aguiar DML, Frazão P. Cooperação interprofissional e a Reforma Sanitária no Brasil: implicações para o modelo de atenção à saúde. Saúde Soc. 2013; 22(1):173-186.

6. Mendes EV. As redes de atenção à saúde. Ciênc. Saúde Colet. 2010; 15(5):2297-2305.

7. Korner M, Butof S, Muller C, et al. Interprofessional teamwork and team interventions in chronic care: A systematic review. J Interprof Care. 2016; 30(1):15-28.

8. Shrader S, Hodgkins R, Laverentz D, K, et al. Interprofessional Education and Practice Guide No. 7: Development, implementation, and evaluation of a large-scale required interprofessional education foundational programme. J Interprof Care. 2016; 30(5):615-619.

9. Reeves S. Porque precisamos da educação interprofissional para um cuidado efetivo e seguro. Interface (Botucatu). 2016; 20(56):185-197.
10. Reeves S. Developing and Delivering Practice-based Interprofessional Education. Berlin: Verlag Dr. Müller; 2008.

11. Zirn L, Körner M, Luzay L, et al. Design and evaluation of an IPE module at the beginning of professional training in medicine, nursing, and physiotherapy. GMS J Med Educ. 2016; 33(2):1-17.

12. Peduzzi M. O SUS é interprofissional. Interface (Botucatu). 2016; 20(56):199-201.

13. Peduzzi M, Norman IJ, Germani ACCG, et al. Educação interprofissional: formação de profissionais de saúde para o trabalho em equipe com foco nos usuários. Rev. Esc. Enferm. USP. 2013; 47(4):977-83.

14. Costa MV, Patrício KP, Câmara AMCS, et al. Pró-Saúde e PET-Saúde como espaços de educação interprofissional. Interface. 2015; 19(supl1):709-720.

15. Silva JAM, Peduzzi M, Orchard C, et al. Educação interprofissional e prática colaborativa na Atenção Primária à Saúde. Rev. Esc. Enferm. USP. 2015; 49(supl2):16-24.

16. Barr H. Interprofessional Education: the genesis of a global movement. United Kingdon: Center For The Advancement of Inteprofessional Care; 2015.

17. Ceccim RB. Equipe de saúde: a perspectiva entre-disciplinar na produção dos atos terapêuticos. In: Pinheiro R, Mattos RA. Cuidado: as fronteiras da integralidade. Rio de Janeiro: Hucitec. 2004; p. 259-278.

18. Organização Mundial da Saúde. Marco para ação em educação interprofissional e prática colaborativa. Genebra: OMS; 2010.

19. Oandasan I, Reeves S. Key elements of interprofessional education. Part 2: Factors, processes and outcomes. J. Interprof. Care. 2005; 19(supl1):39-48.

20. Batista NA, Vilela RQB, Batista SHSS. Educação Médica no Brasil. São Paulo: Cortez; 2015. 
21. Camara AMCS, Pinho DLM. A educação interprofissional no PET-Saúde: uma experiência de educação transformadora. Cad. Educ. Saúde Fisioter. 2015; 2(3):817-829

22. Pan American Health Organization. Interprofessional Education in Health Care: Improving Human Resource Capacity to Achieve Universal Health. Report of the Meeting. Bogota, Colombia, 7-9 Dec 2016. Washington, DC: PAHO; 2017.

23. Brasil. Ministério da Saúde. Relatório Final da Oficina de Alinhamento Conceitual sobre Educação e Trabalho Interprofissional em Saúde. Brasília, DF: Ministério da Saúde; 2017.

24. Brasil. Ministério da Saúde, Secretaria de Gestão do Trabalho e da Educação na Saúde. Departamento de Gestão da Educação na Saúde. Política Nacional de Educação Permanente em Saúde: o que se tem produzido para o seu fortalecimento? Brasília, DF: Ministério da Saúde; 2018.

25. Freire Filho JR, Costa MV, Forster AC, et al. New national curricula guidelines that support the use of interprofessional education in the Brazilian context: an analysis of key documents. J Interprof Care. 2017; 31(6):754-760.

26. Freire Filho, JR, Silva CBG. Educação e prática interprofissional no SUS: o que se tem e o que está previsto na política nacional de saúde. Interprofissionalidade e formação na saúde: onde estamos? Porto Alegre: Rede Unida; 2017.

27. Costa MV, Freire Filho JR, Silva GBG, et al. A Educação e o trabalho interprofissional alinhados ao compromisso histórico de fortalecimento e consolidação do
Sistema Único de Saúde (SUS). Interface (Botucatu). 2018; 22(supl2):1507-1510.

28. Silva FAM, Cassiani SHB, Freire Filho JR. Interprofessional Health Education in the Region of the Americas. Rev. Latino-Am. Enfermagem. 2018; 26:e-3013.

29. Costa MV, Peduzzi M, Freire Filho JR, et al. Educação Interprofissional em Saúde [E-book]. Natal: SEDISUniversidade Federal do Rio Grande do Norte; 2018.

30. Brasil. Ministério da Saúde; Organização Mundial da Saúde no Brasil. Laboratório de Inovação em Educação na Saúde com ênfase em Educação Permanente. Brasília, DF: Ministério da Saúde; 2018. Série. Técnica NavegadorSUS 1.

31. Brasil. Ministério da Saúde, Secretaria de Gestão do Trabalho e da Educação na Saúde. PET-Saúde divulga resultado final dos projetos selecionados [internet]. 2018 [acesso em 25 mar 2019]. Disponível em: http://portalms.saude.gov.br/noticias/sgtes/44588-pet-saude-divulga-resultado-final-dos-projetos-selecionados.

32. Hepp SL, Suter E, Jackson K, et al. Using an interprofessional competency framework to examine collaborative practice. J Interprof Care. 2015; 29(2):131-137.

33. Reeves S. Réplica. Interface (Botucatu). 2016; 20(56):205-206.

Recebido em 11/11/2018

Aprovado em 18/04/2019

Conflito de interesses: inexistente

Suporte financeiro: não houve 